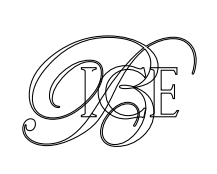

\title{
UNA RESPUESTA A LA PANDEMIA PARA TRANSFORMAR LA ECONOMÍA EUROPEA
}

La crisis económica desatada por la pandemia de la COVID-19 ha supuesto un reto sin precedentes para la Unión Europea. Sin embargo, y a diferencia de lo ocurrido en la anterior crisis, la Unión Europea se ha movilizado para dar una respuesta conjunta que ha logrado articular en unos pocos meses. Esto resulta esencial para garantizar la igualdad de oportunidades de los distintos países en la fase de recuperación, independientemente de su capacidad individual de apoyar sus economías, evitando así la creación de nuevas divergencias. En este artículo se presentan, en primer lugar, las medidas europeas a los retos a corto plazo y, en segundo lugar, se describe el plan de recuperación económica que busca allanar el camino para una recuperación simétrica con una perspectiva de medio plazo. La conclusión fundamental es que la Unión Europea ha sabido estar a la altura del reto, ofreciendo una respuesta rápida y eficiente, aunque persiste la necesidad de abordar reformas profundas en el diseño de la Unión para mejorar su capacidad de respuesta ante futuras crisis.

Palabras clave: COVID-19, Unión Europea, plan de recuperación económica, Comisión Europea, Mecanismo Europeo de Estabilidad, Banco Europeo de Inversiones.

Clasificación JEL: E52, E62, F30, F45, G28.

\section{Introducción}

Mientras que a principios de 2020 se preveía que se mantuviera una tendencia de crecimiento generalizada, aunque en un contexto de cierto agotamiento del ciclo expansivo iniciado en 2013, la irrupción de la pandemia ha dado lugar a la mayor recesión que ha vivido la Unión Europea; una recesión global, con importantes

*Secretaría General del Tesoro y Financiación Internacional, Ministerio de Asuntos Económicos y Transformación Digital.

Este artículo ha sido elaborado por Teresa Abad Hernández, Mercedes Buades Toribio, Carla Díaz Álvarez de Toledo, Víctor García Begué, Marta Martínez Guerra y Enrique Ruiz de Villa Saiz.

Versión de agosto de 2020

DOI: https:/doi.org/10.32796/bice.2020.3127.7088. interrogantes acerca de su magnitud final y la posterior recuperación. Para 2021 se espera una fuerte recuperación en la mayoría de los países europeos, pero es necesario tener presentes los posibles riesgos a la baja, así como el riesgo de que una recuperación asimétrica amplíe las divergencias en la UE, poniendo en peligro el propio proyecto europeo.

Si la contundente respuesta fiscal en un primer momento tuvo un carácter fundamentalmente nacional, desde el principio quedó en evidencia que la respuesta debía ser coordinada, sobre todo a nivel europeo. Frente a un shock exógeno común, es fundamental preservar la igualdad de oportunidades en todos $D$ 
los países europeos para evitar fragmentar el mercado interior. Además, las importantes interconexiones entre las economías europeas implican que si una región se queda atrás en la fase de recuperación, las consecuencias serían negativas para el conjunto de la UE.

Lo cierto es que, aunque el shock haya sido exógeno, el impacto está siendo asimétrico. No solo en términos de la gravedad de la pandemia, sino también en la fase de recuperación, dependiendo de la especialización sectorial de las economías o de su estructura empresarial. Esto requiere que la respuesta coordinada se centre, por tanto, en las regiones y sectores más afectados, contribuyendo efectivamente a paliar el impacto de la crisis. En este sentido, la acción común a nivel europeo está justificada, en particular para restablecer la igualdad de condiciones y evitar divergencias entre los Estados miembros de la UE.

El presente artículo se centrará en la respuesta europea a la crisis, en la que cabe destacar, por un lado, las medidas a corto plazo acordadas en el Eurogrupo, que se componen de tres instrumentos (la línea de crédito del Mecanismo Europeo de Estabilidad, el instrumento SURE y el Fondo Paneuropeo de Garantías del Banco Europeo de Inversiones); y por otro lado, el paquete de recuperación europeo acordado por los líderes políticos en el Consejo Europeo del 21 de julio, un paquete integral de 1,8243 billones de euros que combina el Marco Financiero Plurianual 2021-2027 y un extraordinario esfuerzo de recuperación bajo el instrumento Next Generation EU.

\section{La respuesta más inmediata a la crisis}

Cuando en marzo la pandemia se extendió de forma generalizada en Europa, las autoridades nacionales pusieron en marcha de forma inmediata medidas orientadas a tres objetivos fundamentales: hacer frente a la emergencia sanitaria, apoyar la provisión de liquidez a la economía y proteger el empleo y a trabajadores, autónomos y población vulnerable.

En este contexto, la magnitud de las medidas implementadas puede variar entre los distintos países en función de la capacidad de apoyo público, lo que afecta a las posibilidades de recuperación de las distintas zonas geográficas, generando riesgos de mayores divergencias. Asimismo, las empresas europeas se benefician de garantías de distinta calidad crediticia en función de su localización geográfica, lo que puede tener un impacto sobre su competitividad, contribuyendo a fragmentar el mercado interior, al igual que sucede con el apoyo estatal a compañías para restaurar su solvencia.

La respuesta más inmediata de las instituciones europeas fue proporcionar un marco adecuado para la implementación de medidas nacionales. El objetivo era evitar que una aplicación mecánica de las reglas europeas impidiera a las autoridades nacionales dar la respuesta necesaria. Por ello, en marzo, la Comisión propuso activar la cláusula de escape del Pacto de Estabilidad y Crecimiento, permitiendo a los Estados miembros desviarse temporalmente de la senda de ajuste fiscal establecida para el año 2020 y adoptar así medidas de apoyo a la economía, preservando también la sostenibilidad de las finanzas públicas a medio plazo. A día de hoy se desconoce cuándo se desactivará esta cláusula para volver al proceso de supervisión fiscal habitual, en un contexto en el que la práctica totalidad de los países europeos superan el umbral de déficit del $3 \%$. Si bien hay ciertos Estados miembros que piden una mayor certidumbre sobre este aspecto, la Comisión prefiere no pronunciarse, teniendo $\triangleright$ 
en cuenta la incertidumbre que rodea de momento las previsiones macroeconómicas, y ha dado indicaciones de que esta flexibilidad se mantendrá también en 2021. En otoño, la Comisión evaluará la situación económica.

En segundo lugar, la Comisión adoptó un nuevo marco temporal de ayudas de Estado, flexibilizando las reglas para el apoyo a empresas por parte de los Estados miembros. Este marco, que desde su adopción en marzo se ha modificado hasta en tres ocasiones, regula las condiciones para prestar apoyo público en forma de subvenciones, recapitalizaciones, garantías o préstamos, así como aseguramiento de la exportación y medidas que reducen costes laborales, fiscales y de seguridad social para las empresas. También prevé medidas específicas para fomentar la investigación, prueba y producción de materiales relacionados con el coronavirus. Si bien el apoyo a empresas ha constituido una parte esencial de la respuesta económica a la pandemia, con el fin de evitar que una crisis de liquidez se convierta en una de solvencia, es necesario resaltar que las diferencias entre países, para proporcionar apoyo a sus empresas, entrañan un riesgo significativo de fragmentación del mercado interior.

En tercer lugar, la Comisión propuso flexibilizar la utilización de los fondos comunitarios, liberando la capacidad restante en el presupuesto comunitario para el año 2020 con el fin de apoyar las medidas implementadas por los países frente a la COVID. Ejemplo de ello es la Iniciativa de Inversión en Respuesta al Coronavirus que permite el uso de $\mathbf{3 7 . 0 0 0}$ millones de euros bajo la política de cohesión para hacer frente a las consecuencias de la crisis de la COVID-19, así como la flexibilización del uso de los fondos de la Política de Cohesión para luchar contra esta emergencia; la ampliación del Fondo de Solidaridad para incluir gastos sanitarios derivados de la pandemia (hasta 800 millones para los Estados miembros más castigados); la activación del Instrumento de Apoyo para Emergencias, dotado con 3.000 millones de euros, de los cuales 300 millones serán asignados al programa RescEU para apoyar el aprovisionamiento de equipos sanitarios, o la flexibilización en el uso del Fondo Marítimo y de Pesca y la Política Agraria Común (PAC) para el apoyo a los agricultores.

Otras instituciones reaccionaron igualmente con prontitud, como es el caso de las autoridades monetarias, adoptando medidas contundentes destinadas a contener los costes de financiación y preservar la liquidez. Así, en marzo, el Banco Central Europeo amplió los programas de compras de activos existentes y adoptó el Programa de Compras de Emergencia para la Pandemia (PEPP, por sus siglas en inglés), dotado actualmente con 1,35 billones de euros. Igualmente, para apoyar la liquidez en la economía real, se han flexibilizado ciertos parámetros en los programas de concesión de liquidez a las entidades de crédito, desarrollando además nuevas operaciones de financiación a plazo más largo (PELTRO).

Por lo que se refiere al sistema financiero en particular, las autoridades supervisoras han optado por flexibilizar temporalmente el cumplimiento de algunos requisitos para facilitar a las entidades el mantenimiento de la liquidez y la financiación a la economía real. Así, el Mecanismo Único de Supervisión ha decidido permitir que los bancos operen temporalmente por debajo de sus niveles de capital y liquidez exigidos en el Pilar 2 guidance (colchón de conservación de capital y ratio de cobertura de liquidez), estimando que esta medida liberará, en términos de requerimientos de capital, hasta 120.000 millones de euros y podría llegar a movilizar hasta 1,8 $\triangleright$ 
billones de euros en préstamos a la economía real. Asimismo, se ha concedido flexibilidad a la hora de clasificar los créditos como dudosos (NPL), ofreciendo un mejor tratamiento a aquellos que cuenten con aval público. Cabe también destacar la recomendación del Mecanismo Único de Supervisión a los bancos de no distribuir dividendos y abstenerse de llevar a cabo recompras de acciones dirigidas a remunerar a los accionistas, al menos hasta finales de 2020. También en el marco de la regulación financiera, se han introducido algunos cambios en el ámbito prudencial para facilitar que los bancos mantengan la financiación a hogares y empresas.

En definitiva, en la respuesta más inmediata a la crisis, el coste recae fundamentalmente en los presupuestos nacionales. Cada país estaría asumiendo individualmente los costes de un shock exógeno que tiene un impacto diferenciado entre países, y la capacidad de cada Estado de proporcionar el apoyo adecuado a sus economías y empresas puede terminar por influir sobre las oportunidades de recuperación, entrañando un riesgo de fragmentación del mercado interior. Además, el coste de las medidas financiadas con deuda es diferente para cada país, dadas las distintas condiciones de financiación en el mercado. A menos que se coordine la respuesta, la recuperación puede conducir a una ampliación de las divergencias económicas. Las respuestas nacional y europea constituyen dos caras de una misma moneda y deben estar adecuadamente sincronizadas para reforzarse mutuamente.

\section{La respuesta europea a corto plazo}

Precisamente para atender a esa necesidad de proporcionar una respuesta europea adecuada, el 9 de abril, el Eurogrupo alcanzó un acuerdo sobre tres mecanismos para responder a las necesidades a corto plazo derivadas de la crisis, basados en préstamos y garantías, además de apuntar en ese acuerdo la puesta en marcha de un fondo de recuperación que se analizará más adelante. Los tres instrumentos suponen un paquete de 540.000 millones de euros que crea una triple red de seguridad para trabajadores (instrumento SURE de la Comisión), empresas (Fondo Paneuropeo de Garantías del Banco Europeo de Inversiones) y Gobiernos (línea de crédito del Mecanismo Europeo de Estabilidad).

A través del mecanismo SURE, la Comisión otorgará préstamos a los Estados miembros para financiar esquemas de mantenimiento del empleo o medidas similares, por un importe máximo de 100.000 millones de euros (con un límite de 60.000 millones de euros para los tres mayores receptores). Para ello, la Comisión se endeudará en el mercado y trasladará las condiciones de financiación a los Estados miembros, lo que permitirá homogeneizar las condiciones en las que los países financian estas medidas, solventando en parte el problema de fragmentación financiera. Tras la formalización de las garantías por parte de los Estados miembros para apoyar el endeudamiento de la Comisión (por importe de 25.000 millones de euros), los mismos están remitiendo a la Comisión sus solicitudes de fondos. Los días 24 y 25 de agosto la Comisión publicó las propuestas de cantidades a asignar para los primeros dieciséis países que han solicitado el instrumento por un importe total de 87.300 millones de euros, siendo Italia (27.400 millones de euros) y España (21.300 millones de euros) los principales beneficiarios.

A través de una nueva línea de crédito del Mecanismo Europeo de Estabilidad (Pandemic $\triangleright$ 
TABLA 1

PRINCIPALES MEDIDAS ADOPTADAS A NIVEL UE DESDE EL INCIO DE LA CRISIS

\begin{tabular}{|c|c|c|c|c|c|}
\hline Institución & Propuesta & Fecha & $\begin{array}{c}\text { Estado } \\
\text { de la propuesta }\end{array}$ & $\begin{array}{l}\text { Quién realiza } \\
\text { el gasto }\end{array}$ & $\begin{array}{c}\text { Tipo } \\
\text { de medida }\end{array}$ \\
\hline \multirow{8}{*}{$\begin{array}{l}\text { COMISIÓN } \\
\text { EUROPEA }\end{array}$} & $\begin{array}{l}\text { Iniciativa Europea de Inversión en } \\
\text { Respuesta al Coronavirus }\end{array}$ & \multirow[t]{2}{*}{$3 / 13 / 2020$} & Aprobado & UE & \multirow[t]{2}{*}{ Presupuestaria } \\
\hline & Fondo de Solidaridad & & Aprobado & UE & \\
\hline & Marco temporal de ayudas de Estado & $3 / 19 / 2020$ & Aprobado & $\begin{array}{l}\text { Estados } \\
\text { miembros }\end{array}$ & \multirow{2}{*}{$\begin{array}{l}\text { Flexibilización } \\
\text { de reglas } \\
\text { existentes }\end{array}$} \\
\hline & $\begin{array}{l}\text { Activación de la Cláusula de escape del } \\
\text { Pacto de Estabilidad y Crecimiento }\end{array}$ & $3 / 20 / 2020$ & Aprobado & $\begin{array}{l}\text { Estados } \\
\text { miembros }\end{array}$ & \\
\hline & $\begin{array}{l}\text { Instrumento de ayuda de Emergencia e } \\
\text { iniciativa RescEU }\end{array}$ & \multirow{3}{*}{$4 / 2 / 2020$} & Aprobado & UE & \multirow{3}{*}{ Presupuestaria } \\
\hline & $\begin{array}{l}\text { Flexibilidad en el uso de los Fondos } \\
\text { Estructurales y de Inversión Europeos }\end{array}$ & & Aprobado & UE & \\
\hline & $\begin{array}{l}\text { Propuesta de Instrumento de Apoyo } \\
\text { Temporal para Mitigar los Riesgos de } \\
\text { Desempleo en una Emergencia (SURE) }\end{array}$ & & Aprobado & $\begin{array}{l}\text { Estados } \\
\text { miembros }\end{array}$ & \\
\hline & $\begin{array}{l}\text { 2021-2027 Marco Financiero Plurianual } \\
\text { (+ instrumento NextGenerationEU) }\end{array}$ & $5 / 27 / 2020$ & $\begin{array}{l}\text { Acuerdo del Consejo } \\
\text { Europeo (instrumentos } \\
\text { en proceso de } \\
\text { negociación legislativa) }\end{array}$ & UE & Presupuestaria \\
\hline $\mathrm{BEl}$ & Fondo Paneuropeo de Garantías & $4 / 9 / 2020$ & Aprobado & $\begin{array}{l}\text { Garantías } \\
\text { nacionales } \\
\text { mutualizadas }\end{array}$ & $\begin{array}{l}\text { Préstamos, } \\
\text { participaciones } \\
\text { en equity, } \\
\text { garantías... }\end{array}$ \\
\hline MEDE & Pandemic Crisis Support & $4 / 9 / 2020$ & Aprobado & $\begin{array}{l}\text { Estados } \\
\text { miembros }\end{array}$ & $\begin{array}{l}\text { Préstamos a } \\
\text { EE MM de la } \\
\text { zona euro }\end{array}$ \\
\hline
\end{tabular}

Crisis Support $\mathrm{ECCL}^{1}$ ) se pretende financiar los costes relacionados con la sanidad, el tratamiento o la prevención de la pandemia, por un importe de hasta 240.000 millones de euros, hasta un $2 \%$ del PIB de cada Estado miembro. Este nuevo instrumento supone una adaptación de los instrumentos del Mecanismo Europeo de Estabilidad para hacer frente a este shock concreto, no exigiéndose otra condición más que utilizar los fondos obtenidos para financiar los gastos elegibles. De esta forma, los Estados miembros pueden financiar estos costes en condiciones homogéneas, dado que, de nuevo, se repercuten los costes de financiación del Mecanismo Europeo de Estabilidad junto con un margen de 10 puntos

1 Enhanced Conditioned Credit Line, uno de los dos tipos de líneas precautorias del Mecanismo Europeo de Estabilidad. básicos y otras comisiones de servicio. Está disponible para todos los países de la eurozona desde el 15 de mayo de este año y hasta finales de 2022, si bien, por el momento, ningún país lo ha solicitado.

A través del Fondo Paneuropeo de Garantías gestionado por el Banco Europeo de Inversiones, los Estados miembros aportarán garantías por un importe de 25.000 millones de euros con el objetivo de movilizar financiación por un importe de hasta 200.000 millones de euros. Las empresas de toda la UE pueden así acceder a una garantía de la misma calidad crediticia (AAA), permitiendo paliar en parte el problema señalado anteriormente de fragmentación del mercado interior por la distinta capacidad de las Administraciones nacionales de proporcionar apoyo a $D$ 
sus empresas. Además, este es el único instrumento de los tres que contiene un componente de compartición de riesgos, pues las pérdidas se mutualizarían entre todos los países participantes. A falta de ultimar detalles operativos, se espera que las primeras operaciones con cargo a este fondo empiecen a probarse a principios de otoño.

Estos tres instrumentos proporcionan una respuesta ágil y rápida por parte de la UE, mediante soluciones a corto plazo adaptadas a la naturaleza de la crisis. A diferencia de la crisis anterior, esta vez los Estados miembros compartían el diagnóstico de la crisis, causada por un shock externo, lo que ha facilitado el diseño de estas soluciones. No obstante, los instrumentos acordados se limitan a proporcionar financiación en condiciones homogéneas, incrementando la deuda nacional, y el coste de las medidas sigue recayendo fundamentalmente en los presupuestos nacionales. Si la capacidad de apoyo de cada país es distinta, estas divergencias se acentuarán en la fase de recuperación. Se necesita también un enfoque coordinado para atender las necesidades de la recuperación, con un tamaño suficiente y proporcional al impacto de la crisis, centrado en las regiones y sectores más afectados, y cuyo coste no recaiga exclusivamente en los presupuestos nacionales.

\section{Un plan de recuperación para Europa}

El 23 de abril, tras respaldar las medidas a corto plazo acordadas en el Eurogrupo, el Consejo Europeo encomendó a la Comisión Europea que presentara una propuesta para crear un fondo de recuperación europeo ligado al próximo marco financiero plurianual 2021-2027.
En abril, España contribuyó al debate con una propuesta ambiciosa para este plan de recuperación, basada en apoyo no reembolsable a los Estados miembros para limitar el incremento en la deuda nacional, abogando por un paquete suficientemente significativo para hacer frente a la magnitud de la crisis. Para la financiación del instrumento, España proponía emisiones de deuda perpetua de la UE aprovechando las condiciones acomodaticias de la política monetaria. El interés de la deuda se pagaría con cargo a nuevos impuestos europeos, limitando el aumento de contribuciones nacionales. La distribución de los recursos entre los países se realizaría a través del presupuesto comunitario, siguiendo una clave de reparto que tuviera en cuenta el impacto de la crisis. España abogaba por un desembolso ágil de los fondos, lo más rápido posible a partir de enero 2021, durante dos o tres años, ofreciendo financiación para la reconstrucción y priorizando las transiciones ecológica y digital, así como la autonomía tecnológica europea, en línea con las prioridades de la Comisión, con un énfasis particular en los sectores más afectados por las medidas preventivas, como el turismo o el transporte. La propuesta española también abogaba por un Marco Financiero Plurianual ajustado que apoye una recuperación rápida, sostenible e inclusiva en Europa.

El 27 de mayo, siguiendo el mandato del Consejo Europeo, la Comisión publicó su propuesta para un plan de recuperación europeo, junto con una propuesta para el próximo Marco Financiero Plurianual. Según las estimaciones de la Comisión, el paquete propuesto podría tener un impacto macroeconómico considerable en el crecimiento (aumento del PIB real de la UE de entorno al $1,75 \%$ en 2021 y 2022 , aumentando al $2,25 \%$ en 2024) y en el empleo (creación de dos millones de empleos entre $\square$ 
el 2021 y 2024). Además, este impacto positivo no solo se dirigiría a los principales beneficiarios, sino a la UE en su conjunto, dados los importantes efectos de desbordamiento de las inversiones y la ausencia de efecto desplazamiento de la inversión privada (crowding-out) en un contexto de bajos tipos de interés.

Por el lado de la financiación, la Comisión propuso crear un nuevo instrumento, llamado Next Generation EU, basado en el artículo 122 del Tratado de Funcionamiento de la Unión Europea (TFUE) ${ }^{2}$, que permitiría a la Comisión endeudarse en los mercados por un valor de 750.000 millones de euros, emitiendo bonos con vencimientos comprendidos entre 2028 y 2058. Se trataría de un instrumento de emergencia único, puesto en marcha durante un periodo temporal y utilizado exclusivamente para la respuesta a la crisis y las medidas de recuperación. Los Estados miembros no tendrían que hacer contribuciones adicionales al presupuesto de la UE durante el periodo 2021-2027, ya que los primeros reembolsos tendrían lugar a partir del próximo marco financiero plurianual y durante un periodo prolongado (de 2028 a 2058). La Comisión puso de manifiesto su intención de proponer nuevos recursos propios para hacer frente al repago de esta deuda minimizando el impacto en las contribuciones nacionales.

Por el lado de los gastos, la Comisión propuso que los recursos procedentes de Next Generation EU se destinaran al presupuesto europeo, dotando de 500.000 millones de euros a apoyo no reembolsable y 250.000 millones de euros a préstamos a los Estados miembros. La Comisión proponía reforzar con estos recursos el próximo Marco Financiero Plurianual, incluyendo la creación de nuevos instrumentos para la recuperación.

2 Artículo que permite al Consejo, a propuesta de la Comisión, adoptar medidas de apoyo a un Estado miembro en circunstancias excepcionales, con carácter temporal, informando al Parlamento Europeo.
Con ello se lograría canalizar la inversión hacia donde más se necesita, reforzar el mercado único, intensificar la cooperación en áreas como la salud y la gestión de crisis, y dotar a la UE de un presupuesto para impulsar la transición ecológica y digital. De acuerdo con la propuesta de la Comisión, los fondos de Next Generation EU se canalizarían a través del Marco Financiero Plurianual 2021-2027 hacia tres pilares:

- La mayor parte de los fondos se destinaría a un primer pilar centrado en el apoyo a los Estados miembros para que se recuperen, reparen y salgan fortalecidos de la crisis: (i) un Mecanismo de Recuperación y Resistencia de 560.000 millones de euros, dirigido a financiar planes de reformas e inversiones presentados por los Estados miembros; (ii) un complemento de cohesión de 55.000 millones de euros, disponible a partir de 2020 y que se asignaría en función de la gravedad de la crisis (conocido como REACT-EU); (iii) financiación adicional para el Fondo de Transición Justa (aumento de 30.000 millones de euros) y para el Fondo Europeo Agrícola de Desarrollo Rural (aumento de 15.000 millones de euros).

- El segundo pilar se centraría en incentivar la inversión privada: (i) el Fondo Europeo para Inversiones Estratégicas (EFSI) estaría equipado con un nuevo Instrumento de Apoyo a la Solvencia para atender las necesidades de recapitalización de empresas; y (ii) la capacidad de InvestEU se vería reforzada, incluyendo un nuevo Instrumento de Inversión Estratégica por valor de 31.000 millones de euros.

- El tercer pilar se centraría en fortalecer los principales programas de la UE $D$ 
para reforzar el mercado único. Se destinarían fondos adicionales a una serie de programas nuevos o ya existentes, como EU4Health, RescEU u Horizonte Europa, entre otros. En cuanto a la acción exterior, la Comisión también propone reforzar el instrumento de ayuda humanitaria y aumentar el tamaño del Instrumento de Vecindad, Desarrollo y Cooperación Internacional.

El 17 de julio, los líderes europeos se reunieron en el Consejo Europeo con la intención de alcanzar un acuerdo político. El Consejo se alargó hasta el 21 de julio, día en que por fin los líderes políticos alcanzaron un acuerdo sobre un paquete integral de 1,8243 billones de euros, que combina el Marco Financiero Plurianual 2021-2027 y un extraordinario esfuerzo de recuperación bajo el instrumento Next Generation EU.

El comúnmente denominado fondo de recuperación (Next Generation EU) mantendrá el tamaño de 750.000 millones de euros inicialmente propuesto por la Comisión, si bien, tras el acuerdo, los recursos se repartirán de la siguiente manera: 390.000 millones de euros en forma de subvenciones a los Estados miembros y 360.000 millones de euros en préstamos, con una composición bastante distinta con respecto a la propuesta inicial de la Comisión. Por un lado, se incrementa la dotación destinada a financiar el principal instrumento, el Mecanismo de Recuperación y Resiliencia, con un ligero aumento de las transferencias (de 310.000 a 312.500 millones de euros) y un considerable aumento de los préstamos (que pasan de 250.000 a 360.000 millones de euros). Por otro lado, se reducen algunas partidas financiadas por Next Generation EU: (i) se han recortado los fondos para investigación del programa Horizonte Europa y en las garantías de InvestEU, que en gran parte están destinadas a inversiones estratégicas; (ii) ha desaparecido el Instrumento de Apoyo a la Solvencia, que iba a proporcionar apoyo al capital de las empresas; (iii) la parte del plan destinada a la transición medioambiental ha registrado fuertes recortes, en el segundo pilar de la PAC y en el Fondo de Transición Justa; (iv) se suprimen los recursos destinados al Programa de Salud (EU4Health), así como al apoyo a países terceros, que se cubrirán solo con el presupuesto ordinario.

En total, se estima que España podría llegar a obtener hasta 140.000 millones de euros de los fondos de Next Generation EU, de los cuales 72.700 millones son en apoyo no reembolsable, en el que destacan los 58.640 en apoyo no reembolsable del Mecanismo de Recuperación y Resiliencia. Con cargo a este mecanismo, España también podría obtener unos 67.000 millones de euros en préstamos.

El Mecanismo de Recuperación y Resiliencia es el principal instrumento del paquete de recuperación acordado, ya que absorberá la mayor parte de los recursos de Next Generation EU con 672.500 millones de euros. El apoyo se distribuye de la siguiente manera: 312.500 millones de euros para apoyo no reembolsable (subvenciones) y 360.000 millones de euros en préstamos. Proporcionará apoyo financiero a gran escala a las inversiones y reformas públicas previstas en los planes nacionales de recuperación y resiliencia que elaborarán los Estados miembros, centrándose especialmente en la transición verde y digital y en las prioridades identificadas en el Semestre Europeo. El instrumento, que se basa en el artículo 175 del TFUE (base legal de la política de cohesión), apoyará la recuperación de las regiones más afectadas por la $\triangleright$ 
TABLA 2

COMPARATIVA DE LA PROPUESTA DE LA COMISIÓN EUROPEA Y EL ACUERDO DEL CONSEJO EUROPEO (Miles de millones de euros)

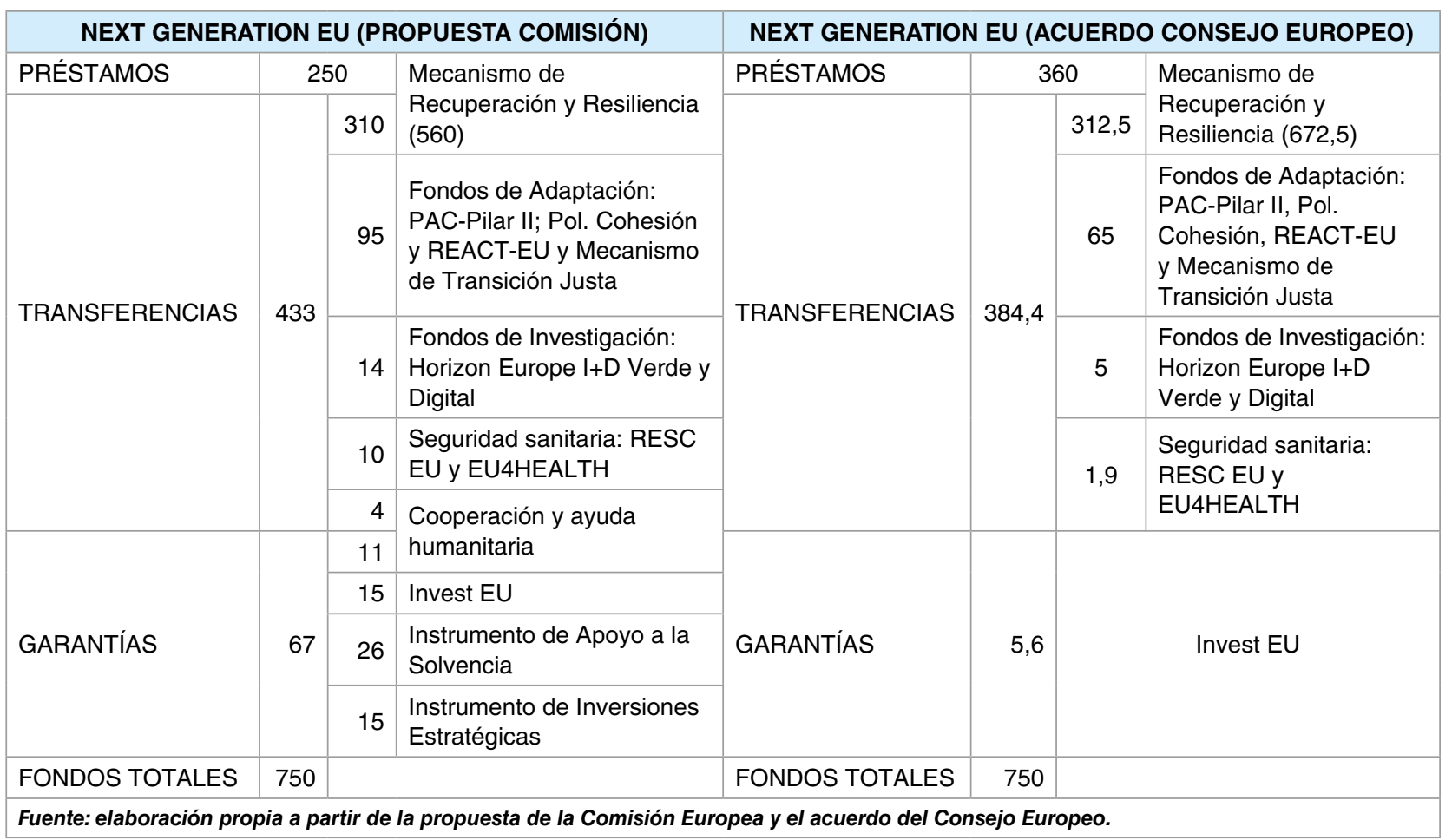

crisis. Sustituye al Instrumento Presupuestario para la Convergencia y la Competitividad (BICC, por sus siglas en inglés) y al Instrumento de Convergencia y Reforma (CRI), que se encontraban en negociaciones legislativas (el BICC se basaba en la idea de crear un presupuesto para los países de la zona euro).

La distribución de los fondos del apoyo no reembolsable, según la clave de reparto acordada en el Consejo Europeo ${ }^{3}$, permite centrar el instrumento en los países más afectados. Los recursos destinados a transferencias

3 El $70 \%$ de los recursos que se asignarán en 2021 y 2022 seguirá la fórmula propuesta por la Comisión en mayo, que tiene en cuenta tres variables: población; inversa del PIB per cápita de 2019, con un tope del $150 \%$ de la media de la UE; y la desviación de la tasa de desempleo media entre 2015-2019 respecto a la media de la UE, con un tope máximo del $150 \%$ de la media de la UE y un tope mínimo del $75 \%$ para países con un PIB per cápita superior a la media de la UE. EI $30 \%$ restante se asignará en 2023, reemplazando el criterio de desempleo de la fórmula de la Comisión por un componente dinámico: la pérdida acumulada de PIB registrada en el periodo 2020 y la registrada en 2020-2021, ponderando cada tasa de variación en un $50 \%$. directas se repartirán de la siguiente manera: un $70 \%$ de los recursos se asignarán en 2021 y 2022, mientras que el $30 \%$ restante se asignará en 2023 para tener en cuenta la caída efectiva de la economía en 2020 y 2021. De esta manera, España podría llegar a obtener alrededor de 58.640 millones de euros en transferencias directas (siendo el segundo país receptor de fondos después de Italia), de los cuales 43.480 millones de euros se asignarían en 2021 y 2022, y alrededor de 15.150 millones de euros se asignarían en 2023. Los países pueden, asimismo, obtener préstamos con cargo a este mecanismo por un importe máximo del $6,8 \%$ de su renta nacional bruta.

En cuanto a la gobernanza del instrumento, aún en negociación, el acuerdo del Consejo Europeo ha modificado los procedimientos con respecto a la propuesta inicial de la Comisión, dando un mayor peso a los países en $\triangleright$ 


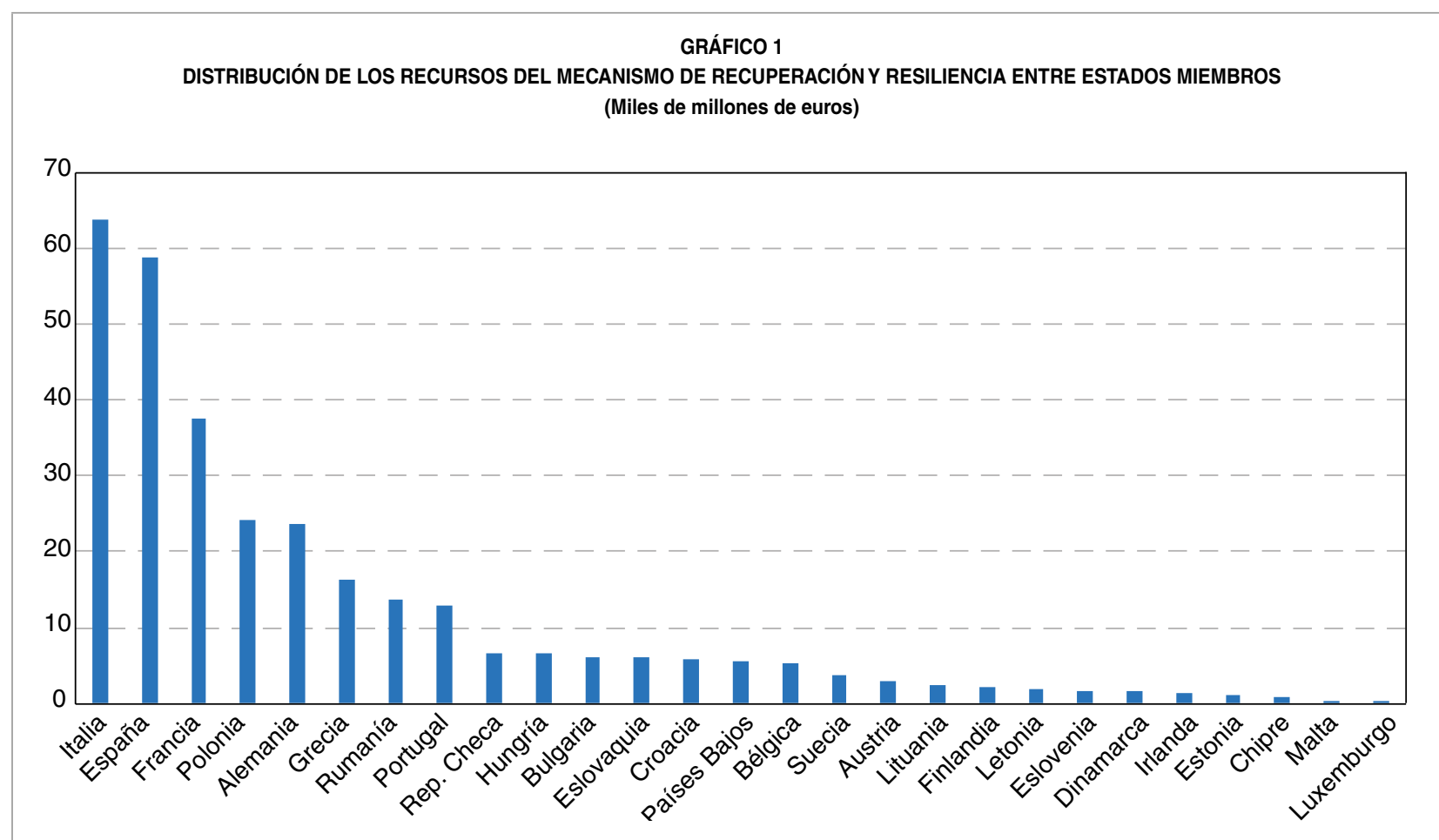

Fuente: elaboración propia a partir del acuerdo del Consejo Europeo, datos de AMECO y previsiones de verano 2020 de la Comisión Europea.

la decisión, tanto para la aprobación de los planes de recuperación y resiliencia (la aprobación tiene lugar en el Consejo por mayoría cualificada, a propuesta de la Comisión) como para la aprobación de los desembolsos para los cuales se incorpora la posibilidad de recurrir al Consejo Europeo si no hay consenso en el Comité Económico y Financiero sobre esta decisión: el acuerdo establece que la Comisión no puede tomar ninguna decisión sobre la aprobación de los desembolsos hasta que «el Consejo Europeo haya examinado esta cuestión en detalle». A este respecto, es esencial evitar un procedimiento que pueda dificultar el desembolso final de fondos y retrasar la necesaria recuperación. Este freno de emergencia debería ser un mecanismo de último recurso destinado a no utilizarse, o solo de forma muy excepcional en casos puntuales y bien justificados, si no se quiere poner en peligro un funcionamiento eficiente del instrumento.
En relación al próximo Marco Financiero Plurianual 2021-2027, el acuerdo alcanzado por los líderes se basa en la propuesta que el presidente del Consejo Europeo presentó a los líderes políticos en febrero (sobre la que no se alcanzó un acuerdo por aquel entonces), adaptada para responder a la crisis de la COVID-19 y a la luz de las medidas adoptadas en el marco de Next Generation EU. De esta manera, el presupuesto de siete años de la UE asciende a 1,074 billones de euros, y se describe en mayor detalle en el primer artículo de esta misma revista (SG de Asuntos Económicos y Financieros, 2020).

Asimismo, los líderes acordaron que, en los próximos años, la Unión trabajará en la reforma del sistema de recursos propios y analizará la introducción de otros nuevos. El producto de los nuevos recursos propios introducidos después de 2021 se utilizará para el reembolso anticipado de los préstamos del Next Generation EU. Como primer paso, se introducirá $D$ 
uno nuevo basado en los residuos plásticos no reciclados y se aplicará a partir del 1 de enero de 2021. Como base para recursos propios adicionales, la Comisión presentará en el primer semestre de 2021 propuestas sobre un mecanismo de ajuste en frontera del carbono, y sobre un gravamen digital, con miras a su introducción a más tardar el 1 de enero de 2023, y trabajará en la introducción de otros recursos propios, entre los que podría figurar un impuesto sobre las transacciones financieras. La introducción de los nuevos recursos propios permitirá hacer frente al repago del endeudamiento derivado del plan europeo de recuperación minimizando el incremento de las contribuciones nacionales.

Sobre la base de la orientación política del Consejo Europeo, el Consejo de la UE y el Parlamento Europeo deben ahora acordar la legislación en que se basan el presupuesto y los programas de financiación sectoriales. El acuerdo alcanzado por el Consejo europeo supone un paso importante, ya que, por primera vez, la Comisión va a emitir deuda en mercados en semejantes cantidades para financiar programas del presupuesto comunitario, apoyando a aquellos Estados miembros más afectados por la crisis. De esta forma, se centraliza parte del coste de la recuperación, y se reparte a lo largo de varios años, lo que resulta adecuado ante la magnitud de la crisis. No obstante, Next Generation EU es un instrumento temporal, sin vocación de permanencia dada su base legal, el artículo 122 del Tratado de Funcionamiento de la Unión Europea, por lo que la idea de crear una verdadera capacidad central de estabilización quedaría descartada por el momento.

Se ha preservado el tamaño total del instrumento Next Generation EU propuesto por la Comisión, 750.000 millones de euros, lo cual es positivo, ya que constituye una respuesta decisiva y contundente fundamental para generar la confianza necesaria para la recuperación. Además, se ha alcanzado un equilibrio razonable entre apoyo no reembolsable y préstamos, una de las cuestiones más controvertidas. La proporción de apoyo no reembolsable (390.000 millones de euros) se ha reducido sustancialmente tras las negociaciones en el Consejo Europeo, pero se mantiene superior al $50 \%$, en contra de lo que habían defendido algunos países, que sostenían que el apoyo debía proporcionarse exclusivamente mediante préstamos (principalmente los cuatro frugales: Países Bajos, Austria, Dinamarca y Suecia).

Dentro del apoyo no reembolsable, se ha preservado íntegramente el Mecanismo de Recuperación y Resiliencia, el principal programa del paquete, incrementándolo sustancialmente, de 560.000 millones de euros a 672.500 millones. De esta forma, se financiarán inversiones y reformas que permitan mejorar el potencial de crecimiento y preserven la convergencia en la UE. No obstante, desaparece el Instrumento de Apoyo a la Solvencia y se reducen otros programas, especialmente los dirigidos a apoyar inversiones, que tenían como objetivo mitigar el impacto de la crisis en la igualdad de oportunidades de las empresas europeas. Esto plantea un reto para la integridad del mercado interior. Así mismo, se reducen los recursos destinados a InvestEU4. De cara al futuro, será fundamental garantizar que InvestEU mantenga una financiación estable en próximos marcos financieros plurianuales, para así atender a las necesidades estructurales de inversión de la UE.

4 Programa de inversiones propuesto para el próximo Marco Financiero Plurianual, que tiene por objetivo dar un impulso adicional a la inversión, la innovación y la creación de empleo en Europa. 
En el acuerdo del Consejo no se precisa con qué recursos se atenderá al repago de la deuda. Aunque este debate puede tener lugar más adelante, pues el horizonte de repago supera el horizonte del próximo Marco Financiero Plurianual, es importante evitar una carga futura excesiva para los países. En este sentido, la creación de nuevos recursos propios, tal como ha adelantado la Comisión, permitirá evitar un incremento significativo de las contribuciones nacionales. No obstante, existen divergencias tradicionales entre los países con respecto a la creación de nuevos recursos propios que será necesario superar.

Por último, se debe señalar la necesidad de que los desembolsos de recursos tengan lugar rápidamente: además del tamaño del programa, es importante que las inversiones empiecen a materializarse cuanto antes y evitar que una crisis de liquidez produzca daños permanentes en la capacidad productiva. Los Estados miembros han logrado alcanzar un acuerdo en julio, lo cual permite sentar las bases para que los instrumentos estén operativos ya desde enero 2021. A diferencia de la crisis anterior, esta vez se ha acordado un programa de recuperación unos meses tras la materialización de la crisis.

\section{Perspectivas de futuro y conclusión}

La crisis actual demuestra que, por muy preparadas que estén las economías, con estructuras flexibles y mecanismos de absorción de crisis, los shocks económicos son inevitables y su magnitud puede sobrepasar los mecanismos de absorción existentes. Lo que marcará la diferencia será la velocidad de recuperación y la medida en que esa recuperación sea inclusiva, evitando dejar legados duraderos. En un contexto en que las economías se encuentran altamente interrelacionadas, es cada vez más importante una respuesta conjunta, evitando divergencias y fragmentación del mercado interior. Se debe ofrecer a todas las regiones las mismas oportunidades de recuperación.

La respuesta europea a la crisis esta vez ha sido rápida y eficiente en cuanto a toma de decisiones y aprobación de instrumentos de apoyo. Ahora bien, será clave que los instrumentos aprobados comiencen a funcionar lo antes posible, y que el plan de recuperación europeo se ponga en marcha sin demoras el 1 de enero de 2021, coincidiendo con el inicio del próximo Marco Financiero Plurianual, con mecanismos eficientes para asegurar desembolsos rápidos. Una de las principales ventajas del paquete de recuperación europeo es que ofrece la oportunidad de impulsar la transformación económica, en particular promoviendo las transiciones verde y digital. Permitirá adelantar inversiones necesarias para modelar las estructuras productivas del futuro y avanzar hacia economías más competitivas y sostenibles en beneficio de las generaciones futuras.

Asimismo, aunque la atención en los últimos meses se ha centrado en las prioridades inmediatas asociadas a esta nueva crisis, junto con la recuperación es fundamental volver a abordar las cuestiones pendientes en la arquitectura financiera europea, de forma que se creen las condiciones para una absorción adecuada de futuros shocks económicos.

En primer lugar, es necesario completar la unión bancaria, dotándola de todas las herramientas adecuadas para abordar una crisis, protegiendo la estabilidad financiera y evitando la fragmentación del mercado comunitario. En esta ocasión, a diferencia de la última crisis, el sector bancario ha sido un factor estabilizador, proporcionando liquidez a la economía real. $\triangleright$ 
Los avances logrados desde la puesta en marcha del Mecanismo Único de Supervisión han permitido que las entidades afronten esta crisis en una posición más robusta. No obstante, esta crisis, en función de su severidad o duración, podría tener un impacto significativo en algunos balances, y es necesario que las instituciones de la unión bancaria cuenten con un mecanismo de gestión de crisis coherente, permitiendo que las entidades de crédito puedan seguir jugando su papel relevante en la recuperación. Ello implica alcanzar un acuerdo sobre un paquete que englobe las cuatro áreas identificadas, en diciembre 2019, por el grupo de trabajo de alto nivel sobre un esquema europeo de garantía de depósitos (EDIS, por sus siglas en inglés): (i) un mecanismo de gestión de crisis coherente para todas las entidades de la unión bancaria, (ii) un esquema europeo de garantía de depósitos que jugaría un papel esencial en este mecanismo de gestión de crisis, (iii) medidas para favorecer la integración del sector bancario en Europa (lo que requiere disponer de redes de seguridad comunes como un EDIS) y (iv) medidas para favorecer la diversificación de exposiciones soberanas en balances acompañadas de la creación de un activo seguro de la zona euro que permita preservar la estabilidad financiera.

En segundo lugar, es necesario finalizar la reforma del Mecanismo Europeo de Estabilidad, cuyo principal objetivo es tratar de reforzar su papel como mecanismo de asistencia financiera, mejorando su capacidad de prevención y corrección de crisis en la eurozona. Los principales puntos contemplados en la reforma son: i) la provisión del backstop del Mecanismo Europeo de Estabilidad al Fondo Único de Resolución a través de una línea de crédito renovable; ii) aumento de la efectividad de los instrumentos precautorios, haciendo más transparentes y predecibles los criterios de elegibilidad; iii) clarificación de las modalidades de cooperación entre el MEDE y la Comisión, tanto dentro como fuera de los programas de asistencia financiera; y iv) mejora del marco de sostenibilidad de la deuda pública, simplificando el sistema de votación en las cláusulas de acción colectiva, o aumentando la transparencia y previsibilidad de la metodología sobre el análisis de dicha sostenibilidad con la publicación de la metodología de las instituciones.

En tercer lugar, la zona euro necesita una capacidad de estabilización central apropiada. Con una política monetaria única, necesita poder implementar un mix de políticas económicas adecuadas. Asimismo, en un contexto de política monetaria muy acomodaticia, la política fiscal debe jugar un mayor papel en la estabilización de las economías.

Además, para acceder a la capacidad de estabilización, los Estados miembros tendrán que garantizar la sostenibilidad de sus finanzas públicas. Esta crisis va a generar incrementos de deuda sustanciales y será necesario diseñar estrategias de consolidación compatibles con el sostenimiento de la recuperación. Por tanto, será necesario un debate sobre la aplicación del Pacto de Estabilidad y Crecimiento en la fase de recuperación, y retomar la discusión sobre la reforma del marco de gobernanza económico europeo, discusión que estaba prevista para principios de este año, pero que, a raíz de la crisis del coronavirus, se ha decidido posponer. En este sentido, sería deseable una mayor predictibilidad y transparencia en las reglas fiscales y en el proceso de supervisión fiscal europeo, mediante la utilización de indicadores observables bajo el control de las autoridades nacionales. Así mismo, sería necesaria una visión más coordinada de las políticas fiscales nacionales, tratando de preservar la sostenibilidad de las $\triangleright$ 
finanzas públicas, sin perder de vista el rol estabilizador de la política fiscal: si bien los Estados miembros con una elevada deuda pública deberían fortalecer su posición fiscal construyendo colchones fiscales, al mismo tiempo se les debería exigir con margen fiscal que impulsaran su crecimiento potencial invirtiendo en infraestructura pública y otros gastos productivos, teniendo en cuenta el escenario actual de bajos tipos de interés. Esto también contribuiría a lograr una posición fiscal agregada adecuada para la zona del euro, fomentando un mejor policy mix con la política monetaria única del Banco Central Europeo.

A la luz de los retos pendientes, sería necesaria una reflexión sobre el futuro de la Unión Económica y Monetaria. Si tras la crisis financiera de 2008 se decidió crear un cortafuegos como el Mecanismo Europeo de Estabilidad y avanzar en un verdadero proyecto común como la unión bancaria, y con la crisis actual provocada por la pandemia se está creando un instrumento común de emisión de deuda (aunque de carácter temporal), de cara al futuro sería conveniente aprovechar las fases de crecimiento económico para dotar a la Unión Económica y Monetaria de una estructura más resistente, sin esperar a que las reformas se vean forzadas por un contexto de crisis.

En conclusión, podría decirse que la Unión Europea ha sabido responder creativamente a las distintas crisis a las que se ha enfrentado, manteniendo la unidad en torno al proyecto común. La crisis actual supone el mayor reto al que se ha visto obligada a hacer frente en su historia. Con una respuesta adecuada, la Unión tiene la oportunidad de sentar las bases que configurarán el futuro del proyecto durante las próximas décadas. Los shocks económicos son inevitables. La Unión Europea debe estar preparada para lo que pueda venir y para ello será crucial que, durante la fase de recuperación venidera, se realice un verdadero esfuerzo de fortalecimiento de la resiliencia de las economías que la conforman y se aborden los retos pendientes para crear una verdadera Unión Económica y Monetaria.

\section{Bibliografía}

Comisión Europea (2020). Propuesta de Reglamento del Consejo relativo a la creación de un Instrumento de Apoyo Temporal para Mitigar los Riesgos de Desempleo en una Emergencia (SURE) a raíz del brote de COVID-19. https:// eur-lex.europa.eu/legal-content/ES/TXT/ $\mathrm{PDF} /$ ?uri=CELEX:52020PC0139\&from $=\mathrm{EN}$

Comisión Europea (2020). Comunicación de la Comisión al Parlamento Europeo, al Consejo Europeo, al Consejo, al Comité Económico y Social Europeo y al Comité de las Regiones. El momento de Europa: reparar los daños y preparar el futuro para la próxima generación. https://eurlex.europa.eu/legal-content/ES/TXT/ $\mathrm{PDF} /$ ?uri=CELEX:52020DC0456\&from=EN

Comisión Europea (2020). Commission staff working document. Identifying Europe's recovery needs. https://ec.europa.eu/info/sites/info/files/ economyfinance/assessment_of_economic_ and_investment_needs.pdf

Consejo de la Unión Europea (2019). Letter by the High-Level Working Group on a European Deposit Insurance Scheme (EDIS) Chair to the President of the Eurogroup. Further strengthening the Banking Union, including EDIS: A roadmap for political negotiations. https://www.consilium. europa.eu/media/41644/2019-12-03-letter-fromthe-hlwg-chair-to-the-peg.pdf

Consejo Europeo (2020). Reunión extraordinaria del Consejo Europeo (17, 18, 19, 20 y 21 de julio de 2020). Conclusiones. https://www.consilium. europa.eu/media/45124/210720-euco-final-conclusions-es.pdf 
Consejo Europeo y Consejo de la Unión Europea. Negotiating the EU long-term budget. https:// www.consilium.europa.eu/en/policies/the-eu-budget/negotiating-the-long-term-eu-budget/

Eurogrupo (2020). Report on the comprehensive economic policy response to the COVID-19 pandemic. https://www.consilium.europa.eu/es/ press/press-releases/2020/04/09/report-on-thecomprehensive-economic-policy-response-to-the-covid-19-pandemic/

Gobierno de España (2020). Spain's non-paper on a European recovery strategy.

Mecanismo Europeo de Estabilidad (2020). Term sheet: ESM Pandemic Crisis Support. https:// www.esm.europa.eu/sites/default/files/20200508pcs-term-sheet-final.pdf
Parlamento Europeo (2020). Nota de prensa: EU summit compromise: positive step for recovery, inadequate in the long-term. https://www.europarl.europa.eu/news/en/press-room/20200721IPR83702/eu-summit-compromise-positive-stepfor-recovery-inadequate-in-the-long-term

Versión Consolidada del Tratado de Funcionamiento de la Unión Europea. Diario Oficial de la Unión Europea, de 26 de octubre de 2012, pp. 326/47 a 326/390. https://eur-lex.europa.eu/legal-content/ ES/TXT/PDF/?uri=CELEX:12012E/TXT\&from=ES

Subdirección General de Asuntos Económicos y Financieros (2020). Marco Financiero Plurianual 2021-2027 y Plan de Recuperación para Europa pos-COVID-19. Boletín de Información Comercial Española (3127), 3-15. 